\title{
Pediatric Multiple Sclerosis in Tunisia: A Retrospective Study over 11 Years
}

\author{
Nedia Ben Achour, ${ }^{1,2}$ Ibtihel Rebai, ${ }^{1}$ Sarra Raddadi, ${ }^{1}$ Hanene Benrhouma, ${ }^{1,2}$ Hedia Klaa, ${ }^{1,2}$ \\ Aida Rouissi, ${ }^{1}$ Ichraf Kraoua, ${ }^{1,2}$ and Ilhem Ben Youssef Turki ${ }^{1,2}$ \\ ${ }^{1}$ Research Unit UR12 SP24 and Department of Child and Adolescent Neurology, National Institute Mongi Ben Hmida of Neurology, \\ Tunis, Tunisia \\ ${ }^{2}$ Faculty of Medicine of Tunis, Tunis El Manar University, 1007 Tunis, Tunisia
}

Correspondence should be addressed to Nedia Ben Achour; nediaachour@yahoo.fr

Received 17 July 2017; Revised 1 October 2017; Accepted 4 October 2017; Published 7 November 2017

Academic Editor: Luca Prosperini

\begin{abstract}
Copyright ( 2017 Nedia Ben Achour et al. This is an open access article distributed under the Creative Commons Attribution License, which permits unrestricted use, distribution, and reproduction in any medium, provided the original work is properly cited.
\end{abstract}

\begin{abstract}
Introduction. Pediatric multiple sclerosis (pMS) is a rare demyelinating disorder with an onset before the age of 18 years. In this study, we aimed to investigate the characteristics of pMS in Tunisian children. Patients and Methods. We conducted a retrospective study over 11 years (2005-2016) including all patients diagnosed with pMS according to the International Pediatric Multiple Sclerosis Study Group (IPMSSG) criteria of 2012 and followed up in a tertiary care research center. Epidemiological, clinical, neuroimaging, laboratory, and therapeutic data were collected and analyzed. Results. There were 21 patients. The male-female ratio was $1: 3$. Mean age at onset was 11 years (range: 3-17 years). Three patients had type 1 diabetes. Polyfocal presentation was preponderant (81\%) with motor dysfunction in $57 \%$ of patients. Paroxysmal dystonia was noticed in $24 \%$. All patients were diagnosed with relapsingremitting form. Interferon beta was prescribed in $80 \%$ with a reduction of annual relapse rate. Conclusion. The annual incidence of pMS in Tunisian children aged below 18 years could be estimated as 0.05 per 100,000. Singular features in our cohort were the frequent association with type 1 diabetes and the increased occurrence of dystonia. Greater awareness of pMS may be helpful to improve management strategies of children and their families.
\end{abstract}

\section{Introduction}

Pediatric multiple sclerosis (pMS) is a rare demyelinating disorder of the central nervous system defined by an onset before the age of 18 years. Diagnosis was based historically on the adult's criteria and children were treated using adult paradigms. It is recognised as multifactorial disease, caused by genetic vulnerability and environmental triggers that are still investigated [1]. In Tunisia, there are no accurate pMS epidemiological data. In this study, we aimed to investigate demographic characteristics, clinical features, biological and radiological aspects, management, and outcome of Tunisian children with pMS.

\section{Patients and Methods}

2.1. Setting. We conducted a retrospective and descriptive study over 11 years (between 1 January 2005 and 31 December
2016) in the Department of Child and Adolescent Neurology at the National Institute Mongi Ben Hmida of Neurology (Tunis, Tunisia). The total population of our country (2005-2016) is $10618933 \pm 399773$, with $95 \%$ confidence interval (CI) of 10.618.863-10.619.002. The pediatric population aged below 18 years (2005-2016) accounts for 3.490.333 \pm 62128 , with $95 \%$ CI of 3.490.314-3.490.352. Our department is a referral tertiary care and research center for children and adolescents with neurological disorders from the whole country and serves a population of over three million people (aged below 18 years). More detailed information about demographics of Tunisia and presentation of our center is provided in Supplementary Material available online at https://doi.org/10.1155/2017/4354826.

2.2. Inclusion Criteria. All patients managed in our department for acquired demyelinating syndromes (ADS) have 


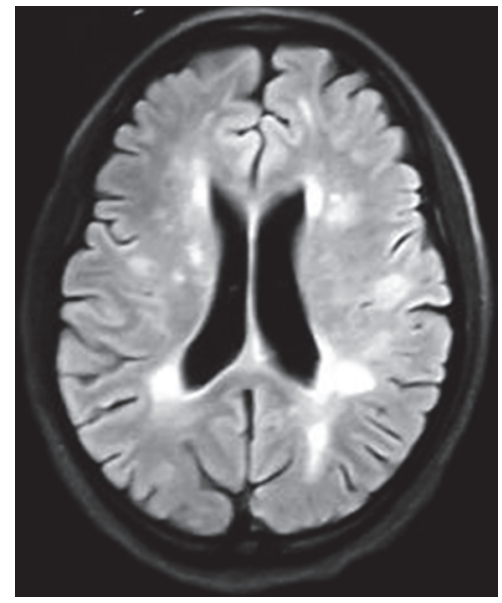

(a)

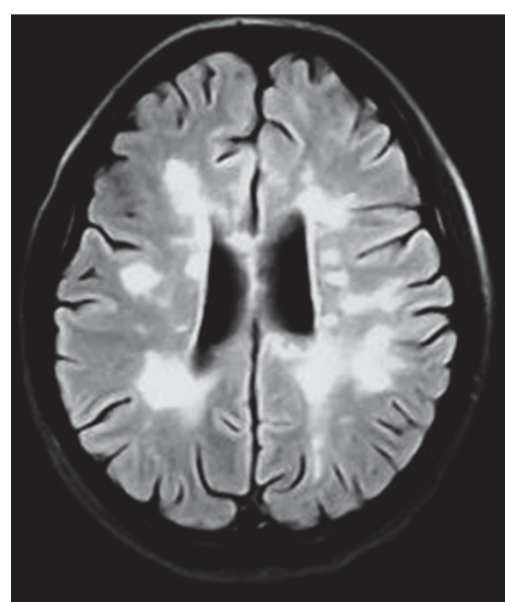

(b)

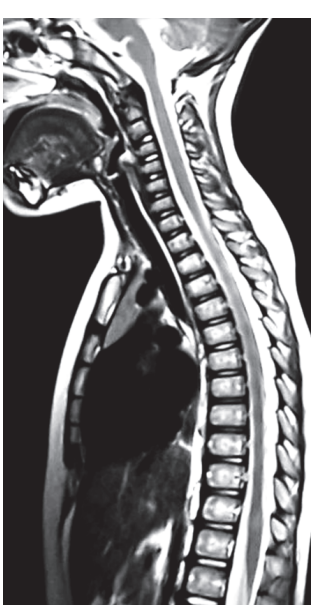

(c)

FIGURE 1: Axial FLAIR weighted images ((a) and (b)) showing multiple hyperintense lesions on periventricular and subcortical regions in patient 5 with spinal dorsal involvement on sagittal T2 weighted image (c).

been retrospectively reviewed. We included in our study all patients who fulfilled the International Pediatric Multiple Sclerosis Study Group's (IPMSSG) revised criteria (2012) [2] for $\mathrm{pMS}$ with a minimum follow-up period $>6$ months.

2.3. Exclusion Criteria. All patients with genetic leukoencephalopathies and demyelinating disorders such as leukodystrophies, aminoacidurias, and mitochondriopathies have been excluded. Patients who presented with acquired demyelinating disorders mimicking MS and whose final diagnoses were infectious, vascular, nutritional (B12 or folate deficiency), or inflammatory disorders (systemic lupus erythematosus, neurosarcoidosis, neuro-Behçet, Sjogren's syndrome,...) have been excluded based on neuroimaging findings and biological and immunological tests. We excluded also all patients with other ADS (CIS, NMO, ADEM,...) who did not fulfill the MS criteria.

2.4. Procedures. Medical records of patients with pMS were retrospectively reviewed. Demographic characteristics, clinical data, Expanded Disability Status Scale (EDSS) score at first consultation and at the last one, biological findings, characteristics of the first magnetic resonance imaging (MRI), and the data about therapeutic management and outcome were collected.

A descriptive analysis was performed using SPSS software.

The study was conducted in accordance with the principles of the Declaration of Helsinki.

\section{Results}

Among 47 patients with ADS, 21 patients fulfilled the diagnosis criteria for pMS and were included in our study. The male-female ratio was $1: 3$. Sixteen patients $(76 \%)$ originated from northern regions, three from the center (14\%), and two from southern regions (10\%).

Mean age at onset was 11 years (range: 3-17 years). Time between the first symptom and the diagnosis of MS varied between 1 day and 2 years.

Medical history corresponded to type 1 diabetes in three cases (10\%) and hypothyroidism, factor 7 deficiency, allergic rhinitis, neonatal jaundice, and hyperpyretic seizures in one case, respectively (4\%).

First onset symptoms occurred in winter and in summer in six patients (28\%), respectively, in autumn in five $(23 \%)$ cases, and in spring in four cases (19\%). The mean follow-up period was three years (ranging from 6 months to 8 years).

Polyfocal presentation was noticed in 17 patients (81\%), whereas monofocal presentation was present in 4 patients (19\%). The most preponderant clinical manifestations were motor dysfunction in 12 patients (57\%), ataxia and brainstem symptoms in 10 patients (48\%), respectively, and sensory dysfunction in 8 patients (38\%). Optic neuritis was documented on visual evoked potentials in 13 patients (62\%). Five patients (24\%) had movement disorders as paroxysmal dystonia. An acute demyelinating encephalomyelitis- (ADEM-) like presentation was noticed in one patient (5\%).

Demographic and clinical data were presented in Table 1.

On their first MRI, all patients showed increased signal on FLAIR and T2 weighted images (Figure 1) with supratentorial lesions in all cases (100\%) and infratentorial involvement in 17 cases $(81 \%)$. Black holes corresponding to hypointense lesions on $\mathrm{T} 1$ weighted images were present in all cases reflecting the axonal injury. Spinal involvement was noticed in 11 cases (52\%) with cervical location in 6 cases $(29 \%)$. Eighteen patients (86\%) had gadolinium enhancement on $\mathrm{T} 1$ weighted images revealing active inflammatory lesions.

CSF analysis was performed in 20 patients (95\%). On their first lumbar puncture, only 8 patients $(38 \%)$ showed positive oligoclonal bands (OCB). An increased CSF cell count (less than 30 lymphocytes $/ \mathrm{mm}^{3}$ ) has been noticed in 5 

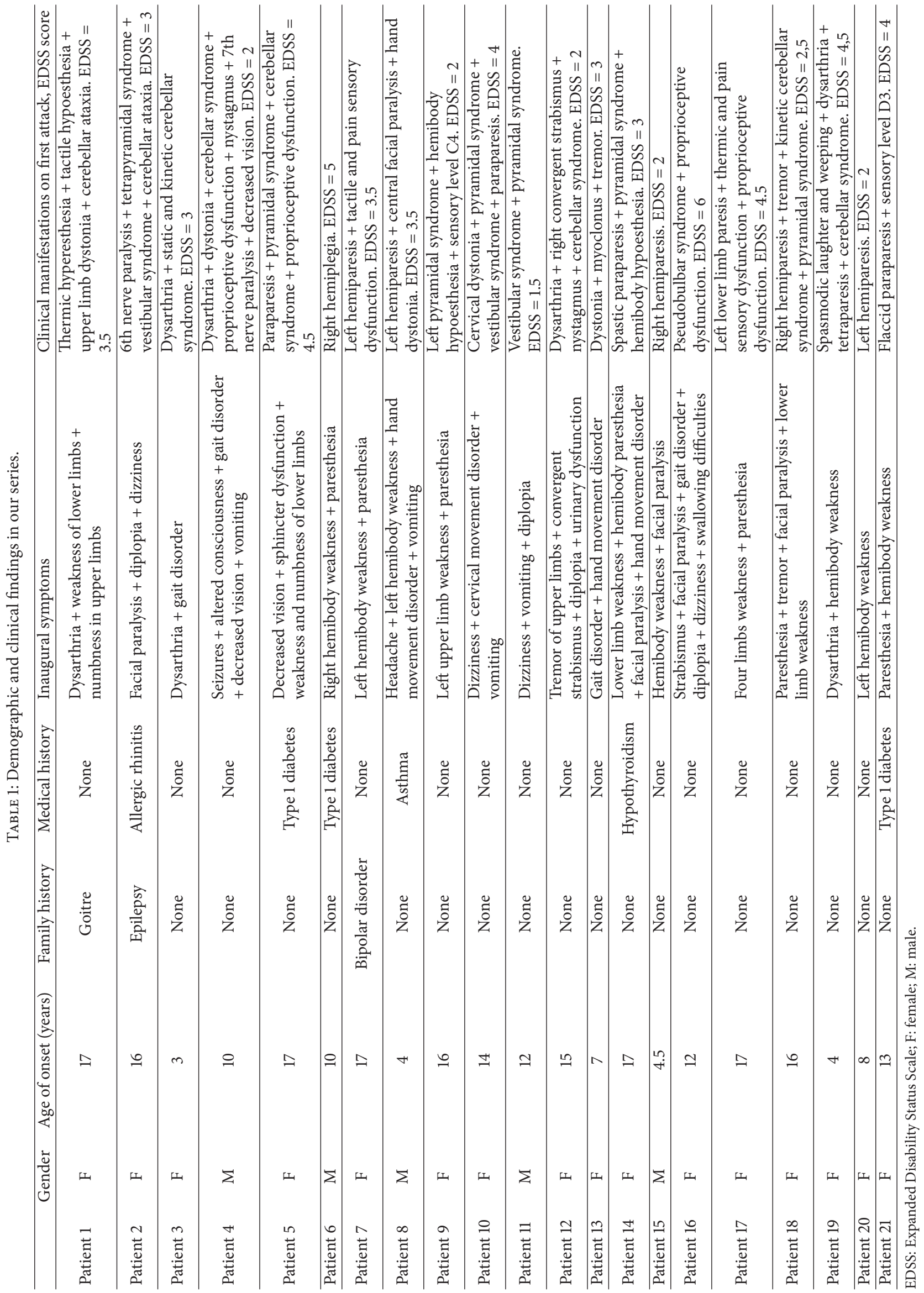
cases (25\%). Patients with negative OCB and/or increased cell count had a second lumbar puncture which revealed positive OCB in $85 \%$.

Detailed neuroimaging and laboratory data were presented in Table 2.

In order to exclude differential diagnosis of MS, all our patients (especially those with increased CSF protein and cell count) had serum and CSF screening for HSV, EBV, VZV, CMV, HIV, rubella virus, hepatitis B and C, mycoplasma, West Nile, HIV, Lyme, and syphilis and showed no argument for recent infection.

Immunological tests (antinuclear, antirheumatoid factor and anti-Sm antibodies) and angiotensin-converting enzyme showed normal results in all patients.

Patients with movement disorders had a screening for Wilson's disease (serum ceruloplasmin test; serum and urine copper tests) and mitochondriopathy (lactate in serum and CSF) and some patients had screening for NMDA antibodies and streptolysin $\mathrm{O}$ antibodies.

Children with a very early onset (patients 3, 8, 15, and 19) had assessment of ammonia, lactate (serum and CSF), amino acid, and urine organic acid chromatography. All these biological investigations showed normal results.

Other biological investigations were performed and aimed to evaluate risk factors for MS. They mainly included measurement of serum 25-hydroxyvitamin D3 (25(OH)D) levels performed in 9 patients. The levels indicated a deficiency state (defined as $25(\mathrm{OH}) \mathrm{D}$ levels less than $10 \mathrm{ng} / \mathrm{ml}$ ) in 6 patients with a lowest rate of $6 \mathrm{ng} / \mathrm{ml}$. Vitamin D insufficiency (defined as 25(OH)D levels between 10 and $20 \mathrm{ng} / \mathrm{ml}$ ) was found in one patient $(18.8 \mathrm{ng} / \mathrm{ml})$.

All patients had been diagnosed with relapsing-remitting MS (RRMS).

Seventeen patients enrolled in the study underwent immunomodulatory therapy with interferon $\beta$ la in 15 cases and interferon $\beta \mathrm{lb}$ in two cases. The EDSS score was 3 at the onset (range: 1.5-6) and 1 at the last evaluation (range: $0-3.5$ ). The median annual relapse rate before and after immunomodulatory therapy was, respectively, 2 (range: $0.25-4$ ) versus 0.42 (range: $0-1.33$ ). Only one patient developed a secondary progressive form.

Therapeutic and follow-up data were presented in Table 3.

\section{Discussion}

Our study provides demographic, clinical, biological, and radiological characteristics, management, and outcome of pMS in Tunisia over 11 years.

Annual incidence of pMS per 100,000 children varies across studies and countries. It has been estimated to be 0.13 in France, 0.18 in Canada, and 0.51 in USA and increased to 2.85 in Sardinia (Italy) $[1,3,4]$. In Tunisia, there are no accurate pMS epidemiological data. Given the demographic characteristics in our country, the annual incidence of pMS in Tunisian children aged below 18 years could be estimated as 0.05 per 100,000 . In patients aged below 15 years, it could be estimated as 0.04 per 100,000 and it increased to 0.08 per 100,000 when considering patients aged between 15 and
18 years. These findings highlight the rarity of pMS in our country $[1,3,4]$ supporting the north-south gradient of MS incidence.

The median age of onset of pMS symptoms in other reported studies ranges between 9.3 and 15.5 years (11 years in our patients) and the median age at diagnosis is about 16 years [1]. A very early onset as found in four of our patients (patients 3, 8, 15, and 19) has been reported [5]. Meanwhile, a very early onset calls to mind the possibility of inherited leukoencephalopathies or inborn neurometabolic disorders and practitioners should be very careful and cautious before retaining the diagnosis of MS.

As shown in our study, pMS is 1.5-2 times more common in female than in male children, suggesting the hormonal pubertal influence on the onset of the disease, whereas the sex ratio is 1 when the disease begins before the age of 10 [6].

A frequent association with type 1 diabetes was found in $14 \%$ of our patients. Both MS and type 1 diabetes are considered as autoimmune disorders with different clinical manifestations. Although pathophysiological mechanisms underlying this association are still unclear, some studies suggest the implication of HLA system particularly the HLADRB1 $* 1501$ allele and the deficiency of vitamin D $[7,8]$.

According to previous reports, 50 to $70 \%$ of patients have a polyfocal presentation as in our series with preponderance of motor dysfunction. Monofocal presentations as optic neuritis or transverse myelitis are less frequent in pMS (around $10 \%)$. ADEM-like presentation is more likely to occur in younger patients displaying polyfocal symptoms with altered behavior or consciousness, which was observed in one patient of our series.

The high occurrence of paroxysmal dystonia (24\%) seems to be a characteristic feature in our patients, whereas it was reported in only $2.9 \%$ of adult MS series and has unknown frequency in the pediatric population [9]. The underlying mechanisms may be related to contiguity of demyelinating lesions to basal ganglia pathways. Interestingly, dystonia was present mainly at onset and was responsive to corticosteroids \pm carbamazepine.

Neuroimaging features of pMS are quite similar to those in adult MS including multiple demyelinating lesions in the periventricular regions, the corpus callosum, and the spinal cord. However, brain lesions in younger children (age < 11 years) may be large and confluent with poorly defined borders at the onset of disease mimicking ADEM lesions. Infratentorial involvement noticed in $81 \%$ of our patients seems characteristic in pMS patients, suggesting the preferential immune targeting of mature myelin $[1,10]$.

CSF analysis, which provides information about the inflammation process, reveals normal cell count and protein level in almost $60 \%$ of cases. Increased CSF cell count less than 30 lymphocytes $/ \mathrm{mm}^{3}$ may be noticed in some children with MS as reported by Sudhakar et al. [10] and as found in our patients. A higher CSF cell count is more suggestive of other diagnoses and requires exclusion of central nervous system infection, vasculitis, autoimmune systemic disorders, or neuromyelitis optica (NMO) [11]. Although OCB are not specific for inflammatory demyelination, they are useful in supporting diagnosis. Sensitivity on initial lumbar puncture 


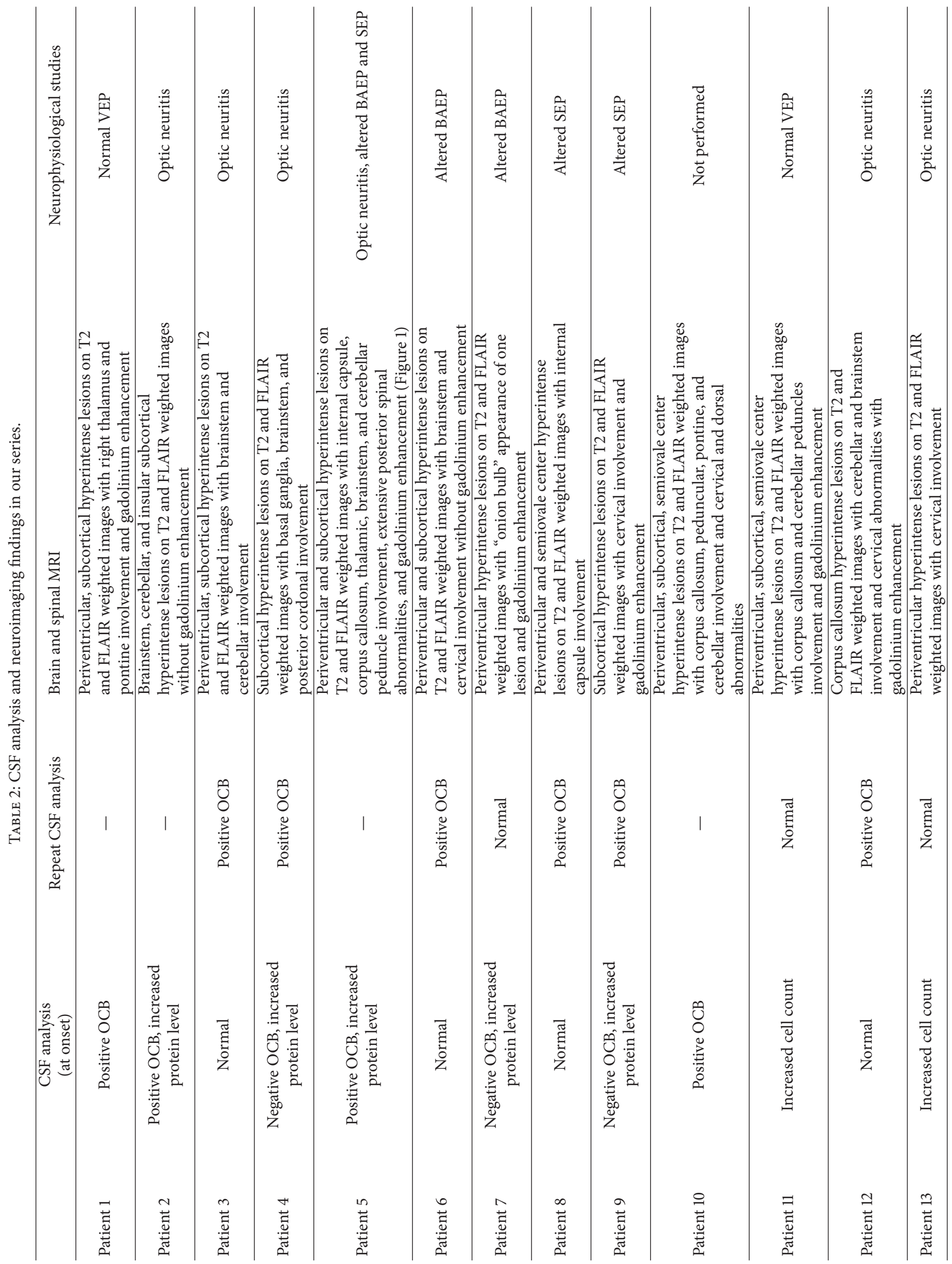




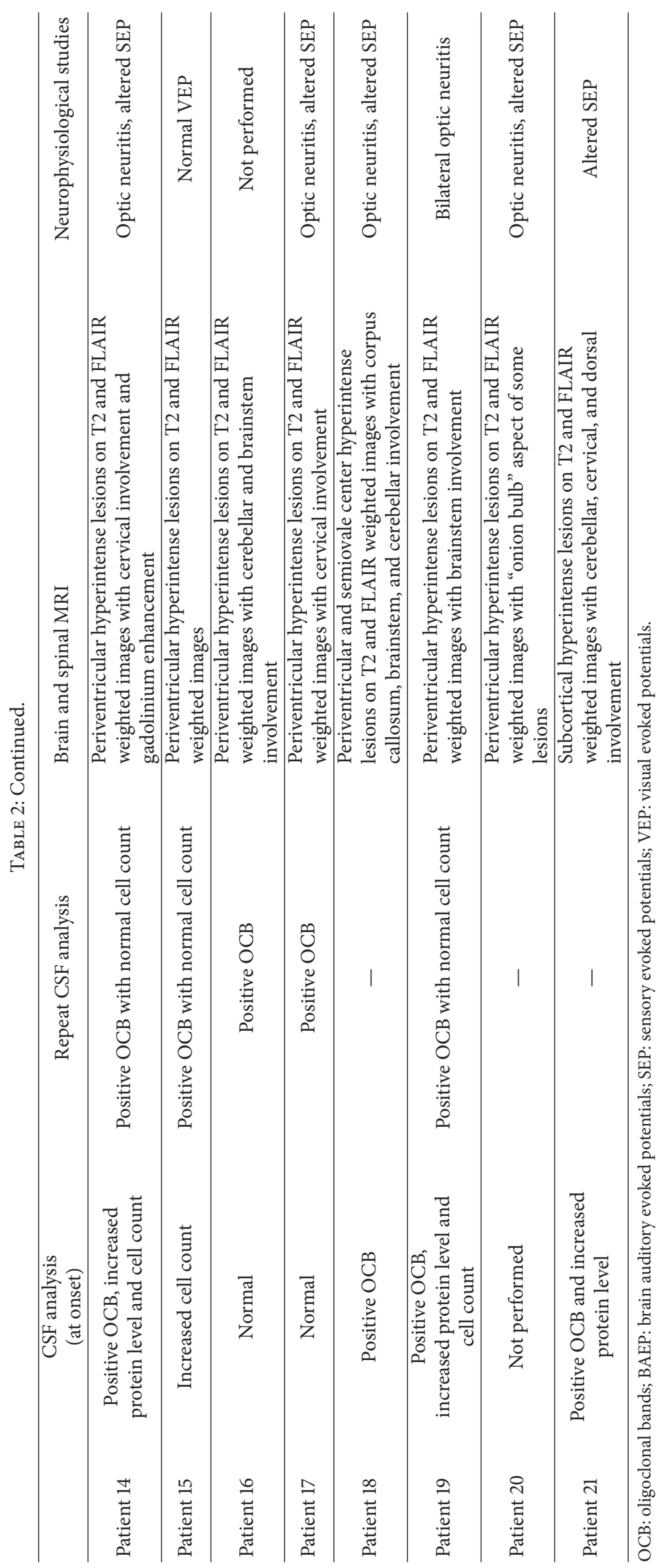


TABLE 3: Therapeutic management and follow-up data in our series.

\begin{tabular}{|c|c|c|c|c|}
\hline & $\begin{array}{c}\text { Immunomodulatory } \\
\text { treatment }\end{array}$ & MS form & $\begin{array}{l}\text { Relapse rate before immunomodulatory } \\
\text { treatment (per year) }\end{array}$ & $\begin{array}{l}\text { Relapse rate after immunomodulatory } \\
\text { treatment (per year) and EDSS score }\end{array}$ \\
\hline Patient 1 & Interferon $\beta$ 1a & RRMS & 2 & $0, \mathrm{EDSS}=1$ \\
\hline Patient 2 & Interferon $\beta$ la & RRMS & 1 & $1, \mathrm{EDSS}=1$ \\
\hline Patient 3 & Interferon $\beta$ 1a & RRMS & 2 & 0, EDSS $=1.5$ \\
\hline Patient 4 & Interferon $\beta$ 1a & RRMS & 1 & $0, \mathrm{EDSS}=1$ \\
\hline Patient 5 & Interferon $\beta$ la & RRMS & 5 & $1.33, \mathrm{EDSS}=1$ \\
\hline Patient 6 & Interferon $\beta \mathrm{lb}$ & RRMS & 1 & $0, \mathrm{EDSS}=0$ \\
\hline Patient 7 & Interferon $\beta$ la & RRMS & 0,5 & $0, \mathrm{EDSS}=1$ \\
\hline Patient 8 & Interferon $\beta$ 1a & RRMS & 3 & 0.3, EDSS $=1$ \\
\hline Patient 9 & Interferon $\beta$ 1a & RRMS & 3 & 0.6, EDSS $=1$ \\
\hline Patient 10 & None & RRMS & - &,- EDSS $=0.5$ \\
\hline Patient 11 & None & RRMS & - &,- EDSS $=0.5$ \\
\hline Patient 12 & Interferon $\beta$ la & RRMS & 2 & 0.33, EDSS $=2$ \\
\hline Patient 13 & None & RRMS & - &,$- \mathrm{EDSS}=1$ \\
\hline Patient 14 & Interferon $\beta \mathrm{lb}$ & RRMS & 2 & $1, \mathrm{EDSS}=1$ \\
\hline Patient 15 & Interferon $\beta$ la & RRMS & 4 & $0.2, \mathrm{EDSS}=1$ \\
\hline Patient 16 & Interferon $\beta$ la & RRMS & 1 & 0.3, EDSS $=1.5$ \\
\hline Patient 17 & Interferon $\beta \mathrm{lb}$ & RRMS & 2 & $1, \mathrm{EDSS}=1$ \\
\hline Patient 18 & Interferon $\beta$ la & RRMS & 4 & $1, \mathrm{EDSS}=1.5$ \\
\hline Patient 19 & $\begin{array}{l}\text { Interferon } \beta \text { la switched } \\
\text { with interferon } \beta \text { lb }\end{array}$ & $\begin{array}{l}\text { RRMS } \\
\text { then SPMS }\end{array}$ & 0.25 & 0, EDSS $=3.5$ \\
\hline Patient 20 & None & RRMS & - &,- EDSS $=1$ \\
\hline Patient 21 & Interferon $\beta$ la & RRMS & 1 & $0.2, \mathrm{EDSS}=1$ \\
\hline
\end{tabular}

RRMS: relapsing-remitting multiple sclerosis; SPMS: secondary progressive multiple sclerosis.

varies between 35\% (young children) and 68\% (adolescent), which is not as high as in adult MS (90\%). As reported in our study, some pMS patients who were OCB-negative at the onset become OCB-positive during follow-up $[1,3,10]$.

To the best of our knowledge, there are no randomized controlled studies regarding the disease modifying therapies in pMS which intend to prevent relapses. Thus, immunomodulatory therapies are derived from adult clinical trials and small retrospective, observational studies.

Interferons or glatiramer acetate is considered as the first-line therapy with proven efficacy and safety [1]. In our series, $81 \%$ of our patients underwent immunomodulatory treatment based on interferon beta with no side effects reported in all of them.

A RRMS form was diagnosed in all patients of our series, which is in agreement with literature, where RRMS form is higher than $80 \%$ in the pediatric population $[3,12,13]$. After the use of modifying therapy, only one patient developed a secondary progressive form.

Consistently with previous reports, a reduction in relapse rate after the initiation of first-line treatments was noticed passing from 2 relapses per year to less than 1 relapse per year. However, some children may present with refractory disease or have poor tolerance and switch to another first- or a second-line therapy as natalizumab, daclizumab, cyclophosphamide, or fingolimod but with an increased risk of side effects when the latter are used which should be balanced against potential benefits. Recent studies report that annual relapse rate seems significantly higher in pMS in comparison with adult MS (1.13 versus 0.4 ), which may be due to the presence of more central nervous system immune cells in the pediatric population with more intense inflammatory reaction, particularly in young children [14].

\section{Conclusion}

Our study illustrates pMS characteristics in Tunisia. The annual incidence of pMS in Tunisian children aged below 18 years could be estimated as 0.05 per 100,000 . Our results are similar to previously reported studies regarding clinical, neuroradiological, and laboratory features which support the different characteristics of pMS compared to the adult form. However, singularity in our cohort was the frequent association with type 1 diabetes as a risk factor for MS and the increased occurrence of dystonia. The underlying mechanisms of pMS are yet to be elucidated requiring further immunobiological research. Thus, greater awareness of this demyelinating disorder may be helpful to better understand the underlying mechanisms and to improve therapeutic and management strategies of children and their families.

\section{Consent}

All parents of patients gave their consent for publication of this data. 


\section{Conflicts of Interest}

The authors declare that there are no conflicts of interest.

\section{Authors' Contributions}

All authors revised the manuscript critically for important intellectual content and gave final approval of the version to be published. Nedia Ben Achour and Ibtihel Rebai contributed equally to this work.

\section{References}

[1] M. Absoud, M. J. Lim, W. K. Chong et al., "Paediatric acquired demyelinating syndromes: Incidence, clinical and magnetic resonance imaging features," Multiple Sclerosis Journal, vol. 19, no. 1, pp. 76-86, 2013.

[2] L. B. Krupp, M. Tardieu, M. P. Amato et al., "International Pediatric Multiple Sclerosis Study Group criteria for pediatric multiple sclerosis and immune-mediated central nervous system demyelinating disorders: revisions to the 2007 definitions," Multiple Sclerosis Journal, vol. 19, no. 10, pp. 1261-1267, 2013.

[3] A. Waldman, A. Ghezzi, A. Bar-Or, Y. Mikaeloff, M. Tardieu, and B. Banwell, "Multiple sclerosis in children: An update on clinical diagnosis, therapeutic strategies, and research," The Lancet Neurology, vol. 13, no. 9, pp. 936-948, 2014.

[4] S. Dell'Avvento, M. A. Sotgiu, S. Manca, G. Sotgiu, and S. Sotgiu, "Epidemiology of multiple sclerosis in the pediatric population of Sardinia, Italy," European Journal of Pediatrics, vol. 175, no. 1, pp. 19-29, 2016.

[5] C. Renoux, S. Vukusic, and C. Confavreux, "The natural history of multiple sclerosis with childhood onset," Clinical Neurology and Neurosurgery, vol. 110, no. 9, pp. 897-904, 2008.

[6] M. Tintoré and G. Arrambide, "Early onset multiple sclerosis: The role of gender," Journal of the Neurological Sciences, vol. 286, no. 1-2, pp. 31-34, 2009.

[7] N. M. Nielsen, T. Westergaard, M. Frisch et al., “Type 1 diabetes and multiple sclerosis: a Danish population-based cohort study," JAMA Neurology, vol. 63, no. 7, pp. 1001-1004, 2006.

[8] P. Tettey, S. Simpson, B. V. Taylor, and I. A. F. Van Der Mei, “The co-occurrence of multiple sclerosis and type 1 diabetes: shared aetiologic features and clinical implication for MS aetiology," Journal of the Neurological Sciences, vol. 348, no. 1-2, pp. 126131, 2015.

[9] R. Mehanna and J. Jankovic, "Movement disorders in multiple sclerosis and other demyelinating diseases," Journal of the Neurological Sciences, vol. 328, no. 1-2, pp. 1-8, 2013.

[10] S. V. Sudhakar, K. Muthusamy, S. Mani, S. Gibikote, and M. Shroff, "Imaging in Pediatric Demyelinating and Inflammatory Diseases of the Brain- Part 1," The Indian Journal of Pediatrics, vol. 83, no. 9, pp. 952-964, 2016.

[11] B. Banwell, L. Krupp, J. Kennedy et al., "Clinical features and viral serologies in children with multiple sclerosis: a multinational observational study," The Lancet Neurology, vol. 6, no. 9, pp. 773-781, 2007.

[12] S. Narula and B. Banwell, "Treatment of multiple sclerosis in children and its challenges," Presse médicale (Paris, France : 1983), vol. 44, no. 4, pp. el53-e158, 2015.

[13] A. Ghezzi, M. P. Amato, N. Makhani, T. Shreiner, J. Gärtner, and S. Tenembaum, "Pediatric multiple sclerosis: Conventional first-line treatment and general management," Neurology, vol. 87, no. 9, pp. S97-S102, 2016.

[14] J. A. Pena and T. E. Lotze, "Pediatric multiple sclerosis: Current concepts and consensus definitions," Autoimmune Diseases, vol. 2013, Article ID 673947, 2013. 


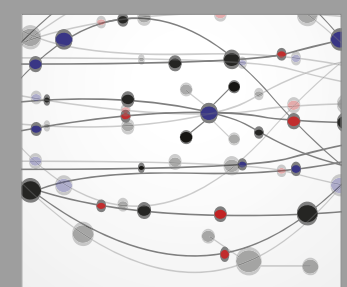

The Scientific World Journal
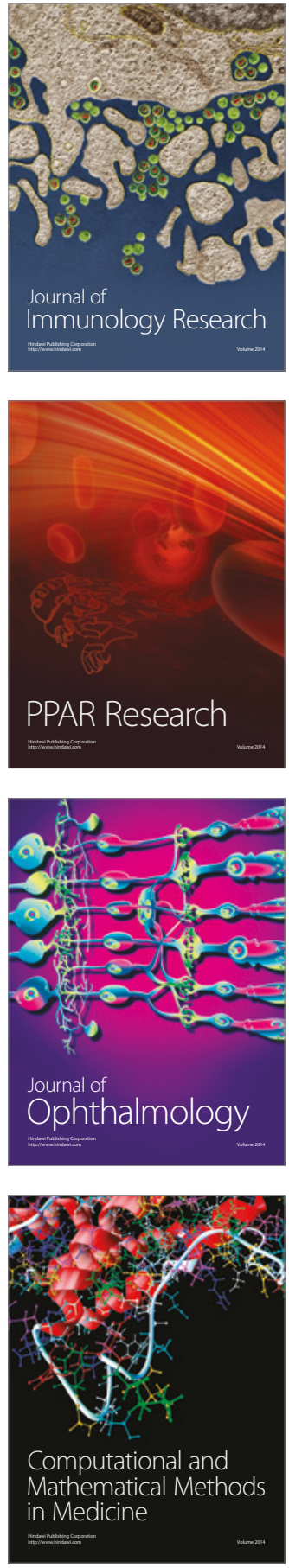

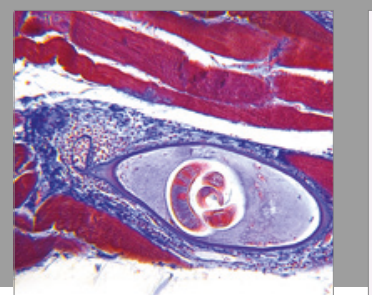

Gastroenterology Research and Practice
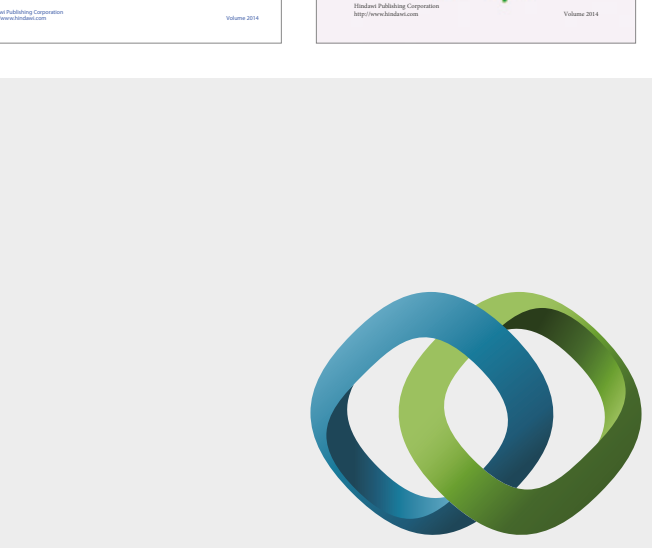

\section{Hindawi}

Submit your manuscripts at

https://www.hindawi.com
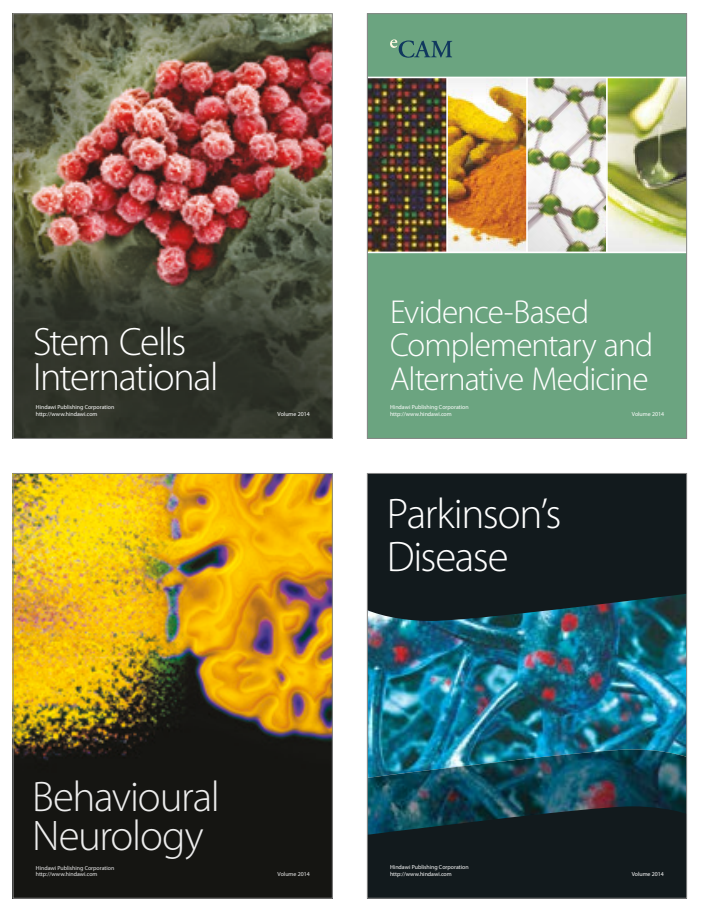
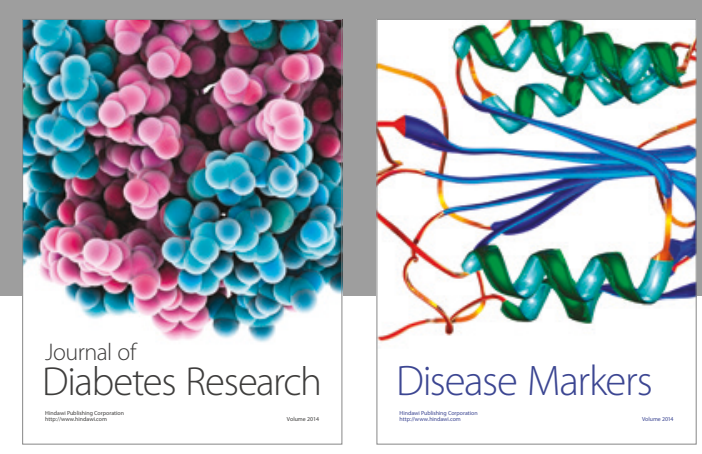

Disease Markers
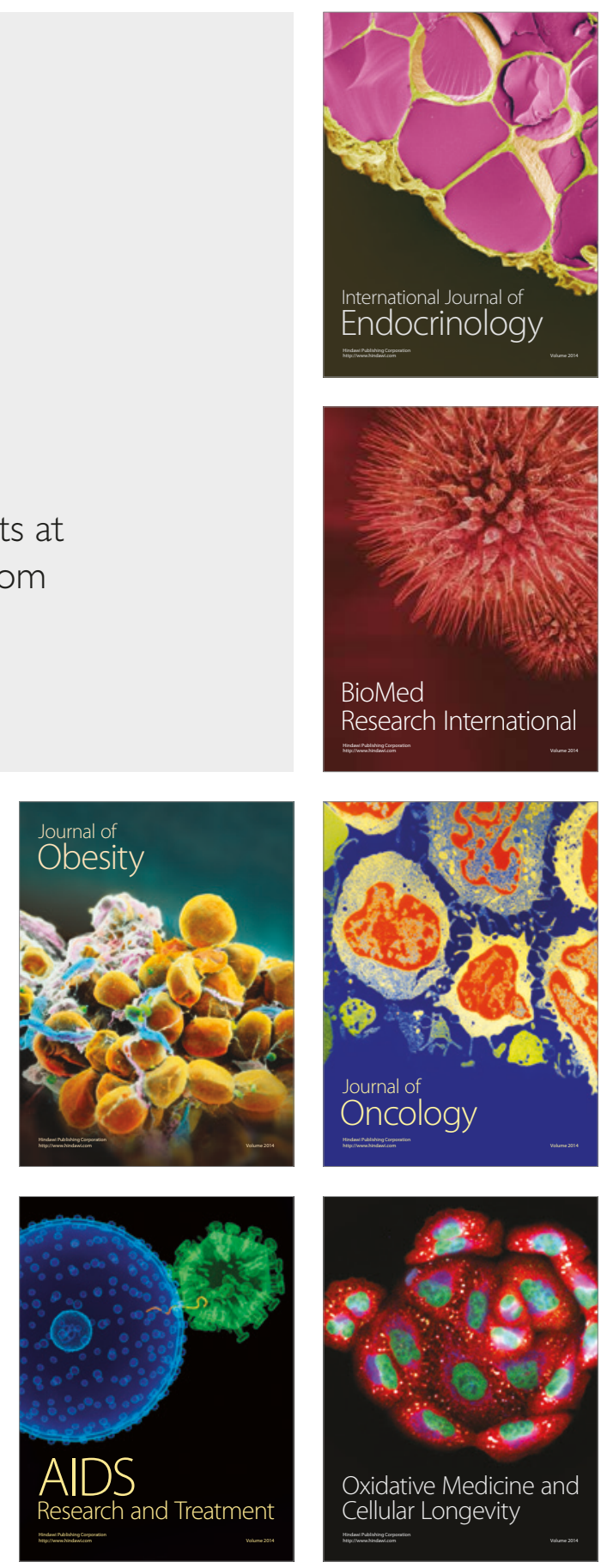